\title{
บUisuersily
}

\section{Effects of Liraglutide and Fenretinide treatments on the diabetic phenotype of neuronal human BACE1 knock-in mice}

Dekeryte, R., Hull, C., Plucińska, K., Khan, S., Kamli-Salino, S., Mody, N., Morrice, N., McLaughlin, C., Gault, V. A., Platt, B., \& Delibegovic, M. (2019). Effects of Liraglutide and Fenretinide treatments on the diabetic phenotype of neuronal human BACE1 knock-in mice. Biochemical Pharmacology, 166, 222-230. https://doi.org/10.1016/j.bcp.2019.05.020

Link to publication record in Ulster University Research Portal

\section{Published in:}

Biochemical Pharmacology

Publication Status:

Published (in print/issue): 01/08/2019

DOI:

10.1016/j.bcp.2019.05.020

\section{Document Version}

Author Accepted version

\section{General rights}

Copyright for the publications made accessible via Ulster University's Research Portal is retained by the author(s) and / or other copyright owners and it is a condition of accessing these publications that users recognise and abide by the legal requirements associated with these rights.

\section{Take down policy}

The Research Portal is Ulster University's institutional repository that provides access to Ulster's research outputs. Every effort has been made to ensure that content in the Research Portal does not infringe any person's rights, or applicable UK laws. If you discover content in the Research Portal that you believe breaches copyright or violates any law, please contact pure-support@ulster.ac.uk. 


\section{Accepted Manuscript}

Effects of Liraglutide and Fenretinide treatments on the diabetic phenotype of neuronal human BACE1 knock-in mice

Ruta Dekeryte, Claire Hull, Kaja Plucińska, Shakil Khan, Sarah Kamli-Salino, Nimesh Mody, Nicola Morrice, Chris McLaughlin, Victor Gault, Bettina Platt, Mirela Delibegovic

PII:

DOI:

Reference:

To appear in:

Received Date:

Accepted Date:
S0006-2952(19)30195-9

https://doi.org/10.1016/j.bcp.2019.05.020

BCP 13533

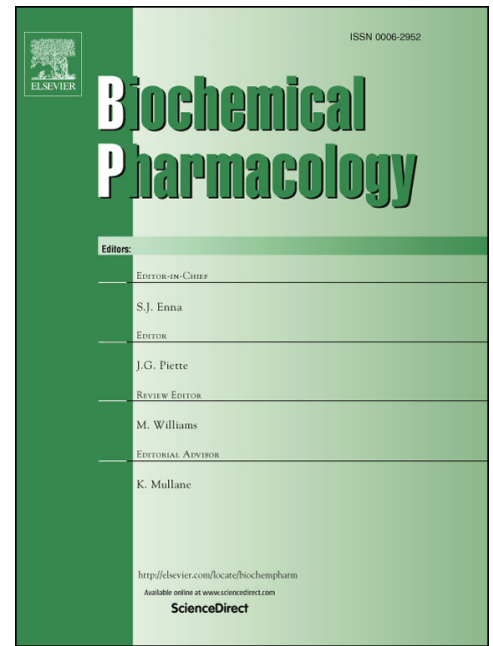

\section{Biochemical Pharmacology}

25 March 2019

14 May 2019

Please cite this article as: R. Dekeryte, C. Hull, K. Plucińska, S. Khan, S. Kamli-Salino, N. Mody, N. Morrice, C. McLaughlin, V. Gault, B. Platt, M. Delibegovic, Effects of Liraglutide and Fenretinide treatments on the diabetic phenotype of neuronal human BACEl knock-in mice, Biochemical Pharmacology (2019), doi: https://doi.org/ 10.1016/j.bcp.2019.05.020

This is a PDF file of an unedited manuscript that has been accepted for publication. As a service to our customers we are providing this early version of the manuscript. The manuscript will undergo copyediting, typesetting, and review of the resulting proof before it is published in its final form. Please note that during the production process errors may be discovered which could affect the content, and all legal disclaimers that apply to the journal pertain. 
Effects of Liraglutide and Fenretinide treatments on the diabetic phenotype of neuronal

\section{human BACE1 knock-in mice}

Ruta Dekeryte ${ }^{1}$, Claire Hull $^{1}$, Kaja Plucińska ${ }^{1,3}$, Shakil Khan ${ }^{1}$, Sarah Kamli-Salino ${ }^{1}$, Nimesh Mody $^{1}$, Nicola Morrice ${ }^{1}$, Chris McLaughlin², Victor Gault ${ }^{2}$, Bettina Platt $^{1 *}$ \& Mirela Delibegovic ${ }^{1 *}$

1. Institute of Medical Sciences, College of Life Sciences and Medicine, University of Aberdeen, Foresterhill Health Campus, Aberdeen AB25 2ZD, UK

2. School of Biomedical Sciences, University of Ulster, Coleraine BT52 1SA, Northern Ireland, UK

3.Present address: NNF Center for Basic Metabolic Research, Integrative Metabolism and Environmental Influences, University of Copenhagen, Blegdamsvej 3B, Mæarsk Tower 7.7, Copenhagen $2200 \mathrm{~N}$, Denmark

*Corresponding authors:

Mirela Delibegovic ${ }^{1}$

m.delibegovic@abdn.ac.uk

Bettina Platt ${ }^{1}$

b.platt@abdn.ac.uk

Category: Metabolic Disorders and Endocrinology 


\section{Abstract}

We recently reported that brain-specific human $\beta$-secretase 1 (BACE1) knock-in (PLB4), a mouse model of 'sporadic' Alzheimer's disease (AD), also develops a severe diabetic phenotype characterised by impaired glucose homeostasis, decreased insulin sensitivity and a fatty liver phenotype. Hence, we here aimed to assess if targeted anti-diabetic therapies (Liraglutide and Fenretinide) would attenuate the diabetic and behavioural phenotype of these mice. PLB4 and wild-type (WT) controls were administered Liraglutide or Fenretinide for ten consecutive weeks alongside vehicle-treated mice. Physiological (body weight and mass composition, glucose tolerance, serum hormone concentration), behavioural (locomotor activity) and molecular assessments were performed in mice pre- and posttreatment. Liraglutide and Fenretinide treatments inhibited adiposity gain and decreased circulating serum triglyceride (with Liraglutide) and leptin (with Fenretinide) levels in PLB4 mice. We also found that PLB4 mice exhibit increased levels of serum dipeptidyl peptidase 4 (DPP4), together with up-regulated hepatic expression of Dpp4, retinol binding protein 4 (Rbp4) and sterol regulatory element-binding 1c (Srebp1c), which was normalised by both treatments. Interestingly, Liraglutide treatment slowed down habituation to a novel environment and increased secondary night activity peak in WT mice, suggesting an impact on circadian activity regulation. However, neither treatment improved glucose homeostasis in PLB4 mice, implying that impaired glucose metabolism in this genotype may not be associated with glucagon like peptide 1 (GLP-1) and/or RBP4-mediated pathways. In summary, this study provides new insights into molecular mechanisms underlying neuronal BACE1-mediated metabolic regulation and implicates BACE1 as a putative regulator of systemic DPP4 levels. 


\section{Keywords}

BACE1; Type 2 diabetes; DPP4; Liraglutide; Insulin resistance.

\section{Introduction}

Investigation into the links between Alzheimer's disease (AD) and type 2 diabetes mellitus (T2DM) has recently gained wide attention. The association between the two disorders has been known for some time, but the mechanistic links remain elusive $[1,2]$. Abnormalities associated with T2DM, such as peripheral hyperinsulinaemia, may lead to decreased cerebral insulin levels, contributing to neuronal loss and acceleration of AD progression [3]. Conversely, we have recently shown that AD-associated brain pathology can induce systemic T2DM-like symptoms [4]. More specifically, we demonstrated that expression of neuronal human $\beta$-secretase 1 (BACE1), the key enzyme in amyloid precursor protein (APP) processing and $A D$ pathology, resulted in a diabetic phenotype prior to AD-like impairments in mice (termed PLB4) $[4,5]$. This was accompanied by neuronal pathology such as endoplasmic reticulum (ER) stress, elevated ceramides and upregulated central and hepatic levels of retinol binding protein 4 (RBP4), an adipokine associated with insulin resistance, likely all working in concert to induce glucose dyshomeostasis in these mice $[4,6]$. Importantly, other studies also confirm the significance of BACE1 in global metabolic regulation [7-9]; altogether implicating

BACE1 as a strong link between AD and T2DM.

Currently, no effective treatment exists for AD. Since insulin sensitivity and/or levels of insulin receptors are often decreased in $A D$ brains $[10,11]$, the use of anti-diabetic agents for treatment of $A D$ may offer an alternative approach. Specifically, compounds that target the glucagon-like peptide 1 (GLP-1) pathway have attracted significant attention; GLP-1 is a glucoregulatory hormone mainly derived from intestinal L-cells in response to food ingestion 
[12]; nevertheless, GLP-1 has been shown to be produced in the brain as well [13]. GLP-1 has a very short circulating half-life ( $2 \mathrm{~min})$ and is rapidly metabolised by dipeptidyl peptidase IV (DPP4) or neutral endopeptidase 24.11 (NEP), converting active GLP-1 into its inactive form [14].

Liraglutide is an approved medication for the treatment of T2DM with an extended half-life ( 13h) compared to endogenous GLP-1, resulting in insulin-sensitizing and glucose lowering effects [15]. However, recent studies have demonstrated that the effects of Liraglutide are not limited to anti-diabetic actions and can target AD-like pathologies as well [16-18]; Liraglutide treatment alleviated oligomeric amyloid $\beta(A \beta)$-induced synaptic loss, phosphorylation of tau and decrease of insulin receptors in the brains of non-human primates [16]. Furthermore, several studies using experimental mouse models of AD demonstrated beneficial effects of Liraglutide against $A D$ pathologies, including decreased $A \beta$ accumulation, tau protein phosphorylation and hippocampal neurodegeneration, as well as improved memory, cognition and motor function [16,19-20]. Collectively, these findings suggested that Liraglutide may be a potential therapeutic agent for both T2DM and AD, and led to currently ongoing clinical trials of Liraglutide in AD patients (ELAD; www.ClinicalTrials.gov identifier NCT01843075). Nevertheless, other in vivo studies demonstrated that chronic Liraglutide treatment has no effect on $A \beta$ expression in transgenic $h A P P_{\text {Lon }} / \mathrm{PS}_{\mathrm{a} 246 \mathrm{E}}$ and hAPPSwe/PS1 $\triangle E 9$ mice, indicating that efficacy of Liraglutide treatment may vary [21].

An alternative therapeutic target may be retinoid receptors, transporters and metabolizers; these have been the subject of considerable ongoing research for obesity, diabetes and AD $[22,23]$. Lately, an interest has been focused on the synthetic retinoid 4hydroxy(phenyl)retinamide (Fenretinide), which has been investigated as a promising 
treatment for obesity and diabetes [24-28]. Although, the precise mechanisms through which Fenretinide mediates its beneficial metabolic effects remains unknown, it was shown to prevent weight gain and leptin oversecretion in high-fat-diet (HFD)-fed mice and improve insulin sensitivity by decreasing adipose and serum RBP4 levels [24], as well as normalise increased fibroblast growth factor 21 (FGF21) levels in diabetic mice [25]. Based on these findings, we hypothesized that Fenretinide may exert beneficial effects in PLB4 mice, as these were found to display RBP4-related hepatic and brain pathology [4].

The aim of this study was to investigate the effects of a ten week treatment with Liraglutide or Fenretinide on comorbid AD- and T2DM-like phenotypes in neuronal BACE1 knock-in (PLB4) and WT controls, in order to assess their therapeutic potential on the AD background.

\section{Materials and methods}

\subsection{Animals}

Animals were housed and tested following European (Directive on the Protection of Animals used for Scientific Purposes, 2010/63/EU) and UK Home Office regulations. Experiments were approved by the University Ethics Board and carried out in accordance with the Animal (Scientific Procedures) Act 1986 and following ARRIVE guidelines. Transgenic PLB4 and WT mice were generated as previously reported [5] and experiments were not blinded to genotype. All animals were group-housed male mice (3-5 mice per cage), $\sim 4$ months when treatment started and $\sim 6$ months old when treatment ended. All mice were $5 \mathrm{~h}$ fasted and afterwards sacrificed by cervical dislocation at the end of the study. 


\subsection{Drug treatments}

Liraglutide was purchased from GL Biochem (Shanghai) Ltd and 0.04\% wt/wt Fenretinide enriched mouse chow diet was purchased from (Research Diets, New Brunswick, NJ) together with chow control diet. Fenretinide was a generous gift of T. Martin (Johnson \& Johnson, New Brunswick, NJ) to N. Mody. WT $(n=26)$ and PLB4 $(n=23)$ mice were randomized and counterbalanced to the three experimental groups (control (CON) WT $n=11$, PLB4 $n=7$ ), Liraglutide (WT n=7; PLB4 n=8) or Fenretinide (WT $n=8$; PLB4 $n=8$ ) according to the body weight. Fenretinide group mice received $0.04 \%$ wt/wt Fenretinide containing chow diet; Liraglutide and CON group mice were administered $25 \mathrm{nmol} / \mathrm{kg}$ Liraglutide or $0.9 \% \mathrm{w} / \mathrm{v}$ saline once-daily i.p. injection five times a week for 10 weeks respectively, and fed chow control diet (Research Diets, New Brunswick, NJ).

\subsection{Body mass composition}

Fat and lean mass composition was analysed using an EchoMRI scanner pre- and at week 7 of treatment in non-fasted mice (EchoMedical Systems, Houston, TX, U.S.A.).

\subsection{Metabolic measurements}

Glucose tolerance tests (GTTs) were performed 7 weeks into treatment following previously described protocol [28]. Briefly, all mice were fasted for $5 \mathrm{~h}$ and tail blood glucose was determined using an AlphaTRAK glucose strips and glucometer (Berkshire, UK) at baseline (time 0) before i.p. injection of glucose ( $2 \mathrm{mg} / \mathrm{g}$ body weight) and at 15, 30, 60 and $90 \mathrm{~min}$ post- injection. 


\subsection{Serum immunoassays}

Tail or trunk blood was collected from $5 \mathrm{~h}$ fasted mice into serum separator Microtubes. Serum was used for insulin, leptin, total GLP-1 and FGF21 determination (Insulin, GLP-1 and FGF21 ELISA, Millipore, Darmstadt, Germany; leptin ELISA, CrystalChem, Zaandam, Netherlands).

\subsection{Triglyceride assay}

Serum triglycerides were determined using calorimetric triglycerides assay in serum from $5 \mathrm{~h}$ fasted mice (triglyceride kit, Sigma Aldrich, Irvine, UK).

\subsection{Immunoblotting}

RBP4 and DPP4 levels were determined in 1 ul of serum from $5 \mathrm{~h}$ fasted animals via immunoblotting protocol as previously reported [29]. Briefly, 1 ul of serum was mixed with 29 ul of $1 x$ SDS-PAGE gel-loading buffer containing $5 \% \beta$-mercaptoethanol, samples heated for $10 \mathrm{~min}$ at $95^{\circ} \mathrm{C}$ and total volume loaded into the $30 \mathrm{ul}$ wells of a pre-cast gel $(4-12 \%$ Criterion XT Bis-Tris, BioRad, USA). Immunostaining was performed using RBP4 (Dako, Glastrup, Denmark; A0040, 1:1000) and DPP4 (Abcam, Cambridge, UK; ab129060, 1:1000) antibodies. Ponceau S (Sigma Aldrich, Irvine, UK) staining was used as a loading control.

\subsection{Gene expression}

Livers were collected from $5 \mathrm{~h}$-fasted animals and snap-frozen in liquid nitrogen. RNA was isolated from frozen liver tissue (right lobe) using TRIzole (Sigma, UK) and cDNA was synthesised from 1 ug RNA using bioscript cDNA synthesis kit (Bioline, UK), oligo (dT) 18 and random hexamer primers. Genes of interest were amplified by quantitative PCR (qPCR), using gene-specific primers and GoTaq qPCR master mix (Promega, Madison, WI, USA). Relative 
expression of hepatic mRNA levels was calculated using the Pfaffl method [30] and normalised to the geometric mean of the three most stable reference genes (NoNo, Ywhaz, 18S).

\subsection{Activity in the PhenoTyper}

Locomotor and circadian activity were assessed using the PhenoTyper home cage observation system (30 cm $\times 30 \mathrm{~cm} \times 35 \mathrm{~cm}$; Noldus IT, Netherlands) with ad libitum access to food and water, following our previous protocol [5]. Habituation activity (mean distance moved during first 3 hours) and post-habituation average hourly activity over $24 \mathrm{~h}$ (mean over five days post-habituation), were considered as parameters of locomotor and circadian activity in mice.

\subsection{Statistical analysis}

Results are expressed as mean \pm SEM. Statistical analyses were performed using one- or twoway ANOVA with post hoc tests or unpaired two-tailed Student's t-test as appropriate. Nonlinear regression analyses (exponential one-phase decay) was applied to analyse the habituation activity in the PhenoTyper. GraphPad Prism 5 software (GraphPad Software, Inc., San Diego, California, USA) was used for analyses. P-values $<0.05$ were considered significant. 


\section{Results}

3.1 Liraglutide and Fenretinide treatments inhibited adiposity gain in PLB4 mice without affecting overall body weight maintenance.

As previously reported [4], PLB4 mice had lower body weight compared to WT controls (Fig. 1a; $\left.F_{(1,160)}=8.7, p<0.05\right)$. Neither Liraglutide nor Fenretinide affected body weights of WT (Fig. 1b) or PLB4 (Fig. 1c) mice during the ten weeks of treatment. Congruent with the lower body weight phenotype, PLB4 mice displayed lower lean mass content compared to WT controls pre- and at week 7 of treatment (Fig. 1d; pre-treatment $p<0.01$; week $7 p<0.0001$ ), and treatments did not affect lean mass in WT or PLB4 mice. However, both Liraglutide and Fenretinide decreased fat mass content in PLB4 mice compared to saline controls (Fig. 1e; Liraglutide: $p<0.05$; Fenretinide: $p<0.01$ ). The relative change in body composition between pre- and post-treatment ( 7 weeks) indicated $\sim 65 \%$ lower fat mass in Fenretinide-treated PLB4 mice compared to PLB4 controls, without significant effects on lean mass (Fig. If and 1g).

\subsection{Impaired glucose tolerance with Fenretinide treatment in BACE1 knock-in mice}

We next assessed glucose homeostasis and in agreement with our previous study [4], PLB4 mice were glucose intolerant (Fig. $\left.1 \mathrm{~h} ; \mathrm{F}_{(1,64)}=18.03, \mathrm{p}<0.001\right)$. Neither treatment affected glucose homeostasis in WT mice (Fig. 1i), similarly to previously described Liraglutide therapy findings in other WT control mice [31]. Liraglutide treatment did not improve glucose homeostasis in PLB4 mice (Fig 1j); surprisingly, however, Fenretinide treatment worsened glucose maintenance in PLB4 mice at 30min post-glucose injection (Fig $1 \mathrm{j} ; \mathrm{p}<0.05$ ), which resulted in an overall increase in glucose excursion during the GTT (Fig $1 \mathrm{k} ; \mathrm{p}<0.05$ ). 


\subsection{Altered serum hormone and lipid levels with Liraglutide and Fenretinide treatments in}

\section{PLB4 mice}

Serum insulin, leptin and triglyceride levels were not affected by treatments in WT mice (Fig. 2a, 2b, 2c). In PLB4, Fenretinide decreased insulin and leptin levels (Fig. 2a, insulin p<0.05; Fig. $2 b$, leptin $p<0.05$ ) and Liraglutide lowered circulating serum triglycerides (Fig. $2 c ; p<0.05$ ). As expected, Liraglutide increased total levels of serum GLP-1 in WT (Fig. $2 d ; p<0.01$ ), but not in PLB4 mice, suggesting that PLB4 mice were unable to respond to Liraglutide's insulinsensitizing effects. Furthermore, a significant decrease in serum FGF21 levels was found in Fenretinide-treated WT mice as compared to controls (Fig. 2e; $p<0.05$ ).

\subsection{Liraglutide and Fenretinide decreased serum RBP4 levels alongside increased serum} DPP4

Elevated levels of circulating and hepatic RBP4 and DPP4 have been linked to impaired glucose homeostasis, insulin resistance and fatty liver phenotype $[6,32,33]$. We have previously shown that PLB4 mice had increased neuronal and hepatic RBP4 protein levels, which suggested that impaired RBP4 secretion and signalling could underlie decreased insulin sensitivity in PLB4 mice [4]. Therefore, we measured serum RBP4 and DPP4 levels in WT and PLB4 mice; whilst no differences were found in serum RBP4 levels in the present cohort of PLB4 mice compared to WT mice (Fig. 3a, 3b), significantly higher levels of serum DPP4 were detected in PLB4 than WT control mice (Fig. 3a, 3b; $p<0.01$ ).

Furthermore, Fenretinide treatment markedly decreased serum RBP4 levels in both WT and PLB4 mice (Fig. 3c, 3d; both genotypes $p<0.0001$ ), which is in agreement with Fenretinide's known mode of action $[6,24,26]$, since RBP4 normally binds the transthyretin (TTR) homotetramer 
while in circulation [29], whereas Fenretinide was shown to disrupt the RBP4/TTR complex leading to increased renal clearance of RBP4 [6]. Liraglutide also caused a small decrease in serum RBP4, which only reached significance in WT but not PLB4 mice (Fig. 3c, 3d; WT $p<0.05$ ).

Intriguingly, Liralgutide and Fenretinide treatments resulted in the opposite effects on serum DPP4 levels compared to serum RBP4; Fenretinide increased serum DPP4 levels in WT mice (Fig. 3e, 3f; $p<0.05$ ) whilst Liraglutide-treated mice exhibited a trend towards increased serum DPP4 levels $(p=0.1)$. In PLB4 mice however, Liraglutide significantly increased serum DPP4 levels (Fig. 3e, 3f; $p<0.05$ ), with no effects of Fenretinide (Fig. 3e, 3f).

\subsection{PLB4 mice displayed up-regulated hepatic Rbp4, Dpp4 and Srebp1c mRNA expression, which was normalised by Liraglutide and Fenretinide treatments}

Since serum RBP4 and DPP4 levels were altered by Liraglutide and Fenretinide treatments, we next investigated hepatic mRNA expression of $R b p 4, D p p 4$ and genes involved in glucose and lipid metabolism. PLB4 vehicle-treated mice presented elevated hepatic Rbp4 and Dpp4 expression compared to WT controls ( $p<0.05$; Fig. 4a, 4b). This was normalised by both, Liraglutide and Fenretinide treatments in PLB4 mice. None of the treatments affected Dpp4 mRNA expression in WT mice. As expected, Fenretinide decreased Rbp4 and Fgf21 expression in WT mice ( $p<0.05$ (Rbp4); $p<0.001$ (Fgf21); Fig. 4a, 4b). Similar changes in Ffg21 expression were also observed in WT mice with Liraglutide treatment ( $p<0.05$; Fig. 4c) without significant alternations in Fgf21 detected in PLB4 mice across the treatment groups, yet, a trend for upregulated Fgf21 expression in Liraglutide-treated PLB4 mice. As expected, Fenretinide treatment specifically led to a significant ( 20 fold) up-regulation of the classic retinoid target 
gene of cytochrome P450 family 26 subfamily A member 1 (Cyp26a1) in both WT and PLB4 mice ( $p<0.001$; Fig. 4d) [24].

Next, we investigated the mRNA levels of genes involved in insulin signalling and gluconeogenesis. PLB4 control mice had down-regulated hepatic insulin receptor (IR) expression ( $p<0.05$; Fig. 4 ), which was increased by Liraglutide and Fenretinide treatments ( $p<0.05$ (Liraglutide); $p<0.001$ (Fenretinide). Fenretinide treatment induced significant upregulation of gluconeogenic genes (glucose 6-phosphatase (G6Pase) and phosphoenolpyruvate carboxykinase (Pepck)) in PLB4 mice ( $p<0.05$; Fig. 4f, 4g), consistent with the worsened glucose clearance phenotype during the glucose tolerance test.

As serum triglycerides were decreased in PLB4 mice with Liraglutide treatment, we also examined mRNA expression of genes involved in lipid regulation. The carbohydrate response element binding protein (Chrebp) was shown to play a major role in mediating lipogenic and glucose-responsive gene transcription [34,35]; Liraglutide treatment decreased the expression of Chrebp in PLB4 mice ( $p<0.05$; Fig. 4h), but it was not changed by any of the treatments in WT mice or Fenretinide-treated PLB4 mice (Fig. 4h). The sterol regulatory element-binding protein 1c (Srebp1c) is one of the main transcription factors regulating de novo lipid synthesis and hepatic lipid homeostasis. PLB4 control liver had significantly higher Srebp1c levels compared to WT control mice ( $p<0.01$; Fig. $4 \mathrm{i})$, which was normalised in both, Liraglutide and Fenretinide treated PLB4 mice ( $p<0.05$; Fig. 4i). Finally, fatty acid synthase (Fas) was down-regulated in WT Fenretinide mice compared to WT controls ( $p<0.05$; Fig. 4j), with no significant alterations found in PLB4 mice with treatments (Fig. 4j). 


\subsection{Altered locomotor activity with Liraglutide and Fenretinide treatments in WT mice}

Finally, we also investigated effects of Liraglutide and Fenretinide in vivo, on habituation to a novel environment, as well as locomotor and circadian activity during week 9 of treatment. PLB4 mice were previously found to have higher initial activity during habituation at 3, 6 and 12 months of age compared to WT controls [5], and the same effect was seen in the present study in PLB4 versus WT control mice ( 6 months of age; Fig. 5a; $F_{(1,324)}=5.46, p<0.05$; curve fit $p<0.0001)$. Although no significant drug effects were detected on habituation activity of WT and PLB4 mice, a significant difference in activity curves between PLB4 control and PLB4 Fenretinide mice was apparent using nonlinear exponential decay analyses (Fig. 5b; $p<0.001$ ). A markedly higher initial (YO) activity was also detected in WT mice on Fenretinide (Fig. 5b; $p<0.0001$ ), suggesting heightened agitation or anxiety in the new surroundings, alongside a strong trend $(p=0.05)$ for increased plateau activity. A trend $(p=0.07)$ for differences in activity curves between WT control and WT Liraglutide mice was also identified (Fig. 5b).

Similar treatment effects on habituation activity in PLB4 mice were also detected; i.e. a delay in habituation was found in Fenretinide-treated PLB4 mice as compared to PLB4 control mice (Fig. $5 c ; p<0.001)$, and a strong trend $(p=0.05)$ for delayed habituation in PLB4 mice on Liraglutide (Fig. 5c).

Overall, no significant differences were found between WT and PLB4 control mice, when assessing ultradian activity (hourly activity over 24 h, mean over five days) (Fig. 5d); however, Liraglutide resulted in a significant increase in a secondary night activity peak $(2 \mathrm{~h}$ prior the end of dark phase period) in WT mice (Fig. 5e; $p<0.05$ ). Although no significant effects of Liraglutide or Fenretinide for overall daily activity in WT or PLB4 mice was found (Fig. 5e, 5f), 
the strong up-regulation of the secondary night activity peak in WT indicates GLP-1 and its receptor involvement in circadian and ultradian activity patterns regulation.

\section{Discussion}

The present study was designed to investigate the effects of Liraglutide and Fenretinide administration in neuronal BACE1 expressing mice (PLB4) in order to assess if pharmacologically altering GLP-1- and/or RBP4-related molecular pathways would be beneficial for diabetes-associated pathologies in AD-prone population with increased neuronal BACE1 expression and/or activity. The major findings of this study demonstrate that both Liraglutide and Fenretinide inhibit adiposity gain and decrease circulating serum leptin (with Fenretinide) and lipid (with Liraglutide) levels under diabetic conditions in transgenic PLB4 mice. However, neither of the treatments improved glucose homeostasis in this genotype, suggesting that impaired glucose metabolism in PLB4 mice may not be associated with GLP-1- and/or RBP4-mediated pathways or was too advanced to benefit from these treatments.

Nevertheless, this study provided valuable new insights into the relationship between neuronal BACE1 and metabolic regulators such as DPP4, showing that T2DM-like phenotype induced by BACE1 knock-in results in increased levels of serum DPP4 alongside elevated expression of hepatic Dpp4 mRNA. A number of recent studies have shown that liver-specific DPP4 promotes systemic insulin resistance, inflammation in the adipose tissue, impaired glycogen storage and fatty liver disease $[32,33,36]$, which resembles the diabetic phenotype of PLB4 mice at 8 months of age [4]. This indicates that an increase in Dpp4 mRNA expression in murine hepatocytes ( 5.5 months old) may possibly influence and/or lead to ectopic lipid 
build-up in the long-term (i.e. increased liver triglycerides at $~ 8$ months of age). This is in agreement with Miyazaki et al., 2012, who reported increased hepatic mRNA and serum levels of DPP4 in patients with non-alcoholic fatty liver disease [37]. Interestingly, both treatments normalised up-regulated hepatic Dpp4, Rbp4 and Srebp1c expression in PLB4 mice, which together with decreased adiposity, suggest an action of both drugs to specifically target lipid-related pathways under diabetic condition of neuronal BACE1 knock-in, without influencing body weight, or importantly, improving glucose homeostasis.

We detected a clear increase in serum GLP-1 levels in Liraglutide-treated WT mice, without changes in serum GLP-1 levels in PLB4 mice, suggesting that endogenous DPP4 or NEP levels and/or activity may be up-regulated in these mice, leading to increased metabolism and/or excretion of GLP-1 analogue, which led to the investigation of serum DPP4 expression. PLB4 control mice had higher levels of serum DPP4 compared to WT mice, which was further increased with Liraglutide treatment. It is known that circulatory and cellular DPP4 functions are different [38], however, only recent studies have started investigating the exact regulatory mechanisms of each. Here, we demonstrate that both, Liraglutide and Fenretinide treatments exerted different effects on soluble and membrane-bound DPP4. A clear increase in serum DPP4 protein levels was detected in WT Fenretinide and PLB4 Liraglutide mice, which may be due to the increase in possible shedding of the membrane-bound DPP4 across the tissues. However, up-regulated hepatic Dpp4 mRNA levels in PLB4 mice were normalised by both treatments, with no appreciable changes detected in WT mice.

Intriguingly, a shift towards higher Fgf21 expression was observed in Liraglutide-treated PLB4 mice liver, which is similar to recent study showing that GLP-1 analogues (liraglutide and 
exenatide 4) increased hepatic transcription, protein and circulating levels of FGF21 while decreasing glucose production in mouse models of diabetes [39]. The authors concluded that GLP-1 triggered hepatic production of FGF21 is required to stimulate glucose lowering effects of GLP-1, silenced via inhibition of FGF21 [39]. Taken together, a tendency for up-regulated, yet, not significantly, hepatic Fgf21, combined with no changes in serum GLP-1 in Liraglutidetreated PLB4 mice and higher circulating DPP4 levels, could perhaps imply that exogenous GLP-1 was degraded prior to completing the stimulation of hepatic FGF21 production and thus, failing to exhibit its glucose lowering effects in these mice. In addition to this, while both treatments down-regulated hepatic Fgf21 expression in WT mice, no metabolic changes were found, suggesting that decreased Fgf21 transcription does not affect glucose homeostasis under normal physiologic conditions.

Regardless of treatment, no significant changes were detected in body weights of WT and PLB4 mice. This could be attributed to normal body weight in WT mice and a lean body phenotype in PLB4 mice since obesity-associated animal models of diabetes were found to be more sensitive to Liraglutide and Fenretinide induced loss in body weight $[24,40]$, with no significant effects observed in normal weight or leaner than WT controls AD mice $[21,41]$.

Data from clinical studies show that heightened plasma DPP4 levels and/or activity are associated with T2DM, obesity and insulin resistant states and are directly correlated with $\mathrm{BMI}$, leptin and insulin levels $[42,43]$. In contrast to this, our data suggest that an increase in circulating DPP4 concentration can be independent of adiposity and body weight gain in mice, since lean PLB4 mice displayed up-regulated serum DPP4 levels, which were further increased 
with treatments alongside decreased fat mass. However, while circulatory DPP4 levels were increased, effects on enzymatic activity of serum DPP4 are unknown.

Recently, it was reported that BACE1 plays a significant role in regulating liver IR cleavage and expression, demonstrating that reduction or increase in BACE1 results in an increase or decrease of hepatic IR protein expression, without changes in mRNA levels [8]. Here, we show that PLB4 mice exhibited lower IR mRNA in the liver, which was markedly raised by both, Liraglutide and Fenretinide treatments. Overall, the decreased hepatic $I R$ expression paired with elevated expression of Rbp4, Dpp4 and Srebp1c in PLB4 mice, imply that neuronal changes induced by BACE1 may regulate hepatic gene expression and contribute to the systemic phenotype in these mice through yet to uncover mechanism, likely involving changes in the hypothalamic/liver axis signalling. In line with our work, a few other studies have also reported that $A D$-associated changes in the brain can trigger peripheral diabetes-like impairments. For example, central infusion of $A \beta$ oligomers induced peripheral glucose intolerance in vivo, linked to pro-inflammatory pathways activation in the hypothalamus, whereas systemic administration did not induce these changes [44].

Neither Fenretinide nor Liraglutide induced any changes in glucose homeostasis in WT mice, confirming a lack of effect of both drugs on systemic glucose regulation under non-diabetic conditions $[12,45]$. However, Fenretinide exacerbated glucose intolerance in diabetic PLB4 mice. This may be due to decreased serum insulin levels in Fenretinide-treated mice, possibly resulting in a reduction of glucose uptake by insulin-sensitive tissues, and/or up-regulated gluconeogenic gene (Pepck and G6ase) expression. In contrast to other studies with T2DMlike models [46-48], we did not detect any changes in serum insulin levels or glucose 
homeostasis and tolerance in PLB4 mice treated with Liraglutide; however, most of the studies investigated obesity-related diabetic animal models and not lean T2DM-like cases, suggesting that a different mechanism may be involved.

Interestingly, Liraglutide resulted in a tendency to slow down habituation and increased secondary night activity peak in WT mice, suggesting an impact on circadian activity regulation. Studies investigating a role for GLP-1 and GLP-1 receptor (GLP-1R) on biological rhythms control have been limited, but several interesting findings have been reported. Human and rodent L-cell activity and GLP-1 secretion was shown to be driven by circadian patterns [49], which can be altered by disruptions in light-dark cycles and obesity-linked dietary alterations $[50,51]$. Administration of the GLP-1 agonist exendin-4 (12 $\mathrm{h}$ before rapidfeeding) was found to affect clock genes transcription in liver and adipose tissue of mice [52]; however, further experiments are required to fully understand the relationship between GLP1 and circadian clock modulation.

In summary, our findings demonstrate that Liraglutide and Fenretinide treatments resulted in decreased adiposity and lipid lowering effects in PLB4 mice and shed new light on BACE1 and DPP4 molecular interconnection. Since neither of the treatments improved glucose metabolism in PLB4 mice, we conclude that T2DM-like phenotype on this background may not be due to alterations in GLP-1 and retinoid-signalling, and/or diabetic pathologies in PLB4 mice were too advanced to benefit from Liraglutide and Fenretinide treatments. 


\section{Acknowledgments}

The authors would like to acknowledge University of Aberdeen PhD studentship to RD and Scottish Alzheimer's Research UK Junior member research grant to RD to perform GLP-1 ELISA and Alzheimer's Research UK grant to BP and MD (ARUK-PG2017B-11). We thank Dr Oliver Helk for advice concerning statistical analyses and Prof Gernot Riedel for kindly providing the PhenoTyper home cages and advice in behavioural studies.

\section{Authors Contributions}

$\mathrm{MD}, \mathrm{BP}$ and $\mathrm{N}$ Mody designed the study; RD carried out the main experimental work, performed the analysis of the data and wrote the manuscript; MD, BP, N Mody and KP critically revised and edited the manuscript. $\mathrm{CH}, \mathrm{KP}$ and $\mathrm{N}$ Morrice assisted with metabolic and behavioural experiments; SK participated in obtaining qPCR data and SKS aided with serum Western Blot experiments; CM and VG advised regarding the DPP4 experiments and revised the manuscript.

\section{Competing Interests}

The authors declare no competing interests. 


\section{Figure legends}

Figure 1. Liraglutide and Fenretinide treatment effects on body weight, body mass composition and glucose homeostasis in WT and PLB4 mice.

(a) Body weights of WT and PLB4 control (CON) mice throughout the 10 weeks of study; comparison of body weight in WT (b) and PLB4 (c) mice across CON, Liraglutide and Fenretinide groups. Lean (d) and fat mass (e) composition in WT and PLB4 mice pre- and at week 7 of treatment. The relative changes (\%) in body mass composition of WT (f) and PLB4 (g) mice. (h) GTT of WT and PLB4 control mice; GTT of WT (i) and PLB4 (j) mice at week 7 of treatment. (k) Total glucose excursion during GTT (Area under curve (AUC) analysis). Number of mice per group: WT CON n=11; WT Liraglutide $n=7$; WT Fenretinide $n=8$; PLB4 CON n=7; PLB4 Liraglutide $n=8$; PLB4 Fenretinide $n=8$. Data expressed as means \pm SEM and significant differences determined using repeated measured two-way ANOVA followed by Bonferroni post hoc test or unpaired two-tailed Student's t-test. Asterisks: ${ }^{*} p<0.05,{ }^{* *} p<$ $0.01, * * * p<0.001, * * * * p<0.0001$.

Figure 2. Effects of Liraglutide and Fenretinide treatments on serum markers.

Comparison of 5 h-fasted insulin (a), leptin (b) and serum triglyceride (c) levels in WT and PLB4 mice treated with Liraglutide or Fenretinide. Total GLP-1 (d) and FGF21 (e) levels in WT and PLB4 serum. Number of mice per group WT CON n=11; WT Liraglutide $n=7$; WT Fenretinide $n=8$; PLB4 CON $n=7$; PLB4 Liraglutide $n=8 ;$ PLB4 Fenretinide $n=8$. Data expressed as means \pm SEM and significant differences determined using two-way ANOVA followed by unpaired two-tailed Student's t-test: ${ }^{*} p<0.05,{ }^{* *} p<$ 0.01 . 
Figure 3. Fenretinide decreased serum RBP4 levels and increased circulating DPP4 in mice.

(a) Representative immunoblots of serum RBP4 and DPP4 in WT versus PLB4 control mice, showing an increase in serum DPP4 levels in PLB4 CON versus WT CON mice. (b) Quantified data of serum RBP4 and DPP4 in WT CON and PLB4 CON mice. Immunoblots representing Liraglutide and Fenretinide treatment effects on serum RBP4 (c) and DPP4 (e) in WT and PLB4 mice; quantification of serum RBP4 (d) and DPP4 (f) levels in WT and PLB4 mice. Number of mice per group: ( $\mathbf{a}$ and $\mathbf{b}$ ) WT CON n=6; PLB4 CON n=6. (c and d) WT CON n=6; WT Liraglutide $n=5$; WT Fenretinide $n=5$; PLB4 CON $n=4$; PLB4 Liraglutide $n=6$; PLB4 Fenretinide $n=7$. Data expressed as means \pm SEM and significant differences determined using one-way ANOVA with post hoc test: ${ }^{*} p<0.05,{ }^{* *} p<0.01,{ }^{* * *} p<0.001,{ }^{* * * *} p<0.001$.

Figure 4. Changes in hepatic transcription of Rbp4, Dpp4 and genes related to glucose and lipid homeostasis.

Quantitative gene expression analysis of hepatic mRNA expression in WT and PLB4 mice revealed a significant increase in Rbp4 (a) and Dpp4 (b) gene expression in PLB4 CON mice compared to WT CON, which was normalised with Liraglutide and Fenretinide treatments. (c) Fgf21 expression was decreased in WT mice on both treatments, but not in PLB4 mice. (d) Cyp26a1 expression was markedly increased on Fenretinide treatment in both groups of mice. (e) IR expression was lower in PLB4 CON mice liver compared to WT CON and increased with both treatments in PLB4 mice. ( $\mathbf{f}$ and $\mathbf{g}$ ) G6Pase and Pepck expression was higher in PLB4 mice treated with Fenretinide. (h) Chrebp gene expression was decreased in Liraglutide-treated PLB4 mice. (i) Hepatic Srebp1c gene expression was elevated in PLB4 CON versus WT CON mice and was normalised with Liraglutide and Fenretinide treatments. (j) Fas expression was down-regulated in Fenretinide-treated WT mice, but was not changed by any of the treatments in PLB4 mice. Gene expression was normalised to NoNo housekeeping gene. Number of mice per group: WT CON n= 8-9; WT Liraglutide $n=6-7$; WT Fenretinide $n=5-7$; PLB4 CON $n=6-7$; PLB4 Liraglutide $n=6-7 ;$ PLB4 Fenretinide $n=6-7$. Data expressed as means \pm SEM and significant 
differences determined using two-way ANOVA followed by two-tailed Student's t-test. Asterisks: *p < $0.05, * * p<0.01, * * * p<0.001$

\section{Figure 5. Liraglutide and Fenretinide treatments altered locomotor activity in mice}

(a) Representative graphs of habituation activity (10 min bins) in WT versus PLB4 control mice, and WT (b) and PLB4 (c) mice across the treatment groups. Post-habituation average $24 \mathrm{~h}$ daily (ultradian) activity in hourly bins over 5 consecutive days in the PhenoTyper of WT versus PLB4 control mice (d). Liraglutide and Fenretinide treatment effects on the daily activity in WT (e) and PLB4 (f) mice during the week 9 of treatment. Number of mice per group: WT CON n=9; WT Liraglutide n=6; WT Fenretinide $n=7 ;$ PLB4 CON n=7; PLB4 Liraglutide $n=8$; PLB4 Fenretinide $n=7$. Data expressed as means \pm SEM and data analysed using nonlinear exponential decay analyses $(a, b, c)$ or repeated measured two-way ANOVA followed by Bonferroni post hoc test $(\mathrm{a}, \mathrm{d}, \mathrm{e}, \mathrm{f})$ : $*_{\mathrm{p}}<0.05,{ }^{*} \mathrm{p}<\mathrm{0.01;} * * * \mathrm{p}<0.001$; $* * * * \mathrm{p}<0.0001$. Parameters: YO (Y value when the time is zero); $\mathrm{K}$ (rate constant). 


\section{References}

1. Janson J, Laedtke T, Parisi JE, O'Brien P, Petersen RC, Butler PC. Increased risk of type 2 diabetes in Alzheimer disease. Diabetes 2004 Feb;53(2):474-481.

2. Li X, Song D, Leng SX. Link between type 2 diabetes and Alzheimer's disease: from epidemiology to mechanism and treatment. Clin Interv Aging 2015 Mar 10;10:549560.

3. Bosco D, Fava A, Plastino M, Montalcini T, Pujia A. Possible implications of insulin resistance and glucose metabolism in Alzheimer's disease pathogenesis. J Cell Mol Med 2011;15(9):1807-1821.

4. Plucińska K, Dekeryte R, Koss D, Shearer K, Mody N, Whitfield PD, et al. Neuronal human BACE1 knockin induces systemic diabetes in mice. Diabetologia 2016;59(7):1513-1523.(36)

5. Plucinska K, Crouch B, Koss D, Robinson L, Siebrecht M, Riedel G, et al. Knock-in of human BACE1 cleaves murine APP and reiterates Alzheimer-like phenotypes. J Neurosci 2014 Aug 6;34(32):10710-10728.

6. Yang Q, Graham TE, Mody N, Preitner F, Peroni OD, Zabolotny JM, et al. Serum retinol binding protein 4 contributes to insulin resistance in obesity and type 2 diabetes. Nature 2005;436(7049):356.

7. Meakin PJ, Harper AJ, Hamilton DL, Gallagher J, McNeilly AD, Burgess LA, et al. Reduction in BACE1 decreases body weight, protects against diet-induced obesity and enhances insulin sensitivity in mice. Biochem J 2012;441(1):285-295.

8. Meakin PJ, Jalicy SM, Montagut G, Allsop DJ, Cavellini DL, Irvine SW, et al. Bace1dependent amyloid processing regulates hypothalamic leptin sensitivity in obese mice. Scientific reports 2018;8(1):55.

9. Meakin PJ, Mezzapesa A, Benabou E, Haas ME, Bonardo B, Grino M, et al. The beta secretase BACE1 regulates the expression of insulin receptor in the liver. Nature communications 2018;9(1):1306.

10. Griffith CM, Eid T, Rose GM, Patrylo PR. Evidence for altered insulin receptor signaling in Alzheimer's disease. Neuropharmacology 2018;136:202-215.

11. Frölich L, Blum-Degen D, Bernstein H, Engelsberger S, Humrich J, Laufer S, et al. Brain insulin and insulin receptors in aging and sporadic Alzheimer's disease. J Neural Transm 1998;105(4-5):423-438.

12. Ørskov L, Holst JJ, Møller J, Ørskov C, Møller N, Alberti KGMM, et al. GLP-1 does not acutely affect insulin sensitivity in healthy man. Diabetologia 1996;39(10):1227-1232.

13. Vrang N, Larsen PJ. Preproglucagon derived peptides GLP-1, GLP-2 and oxyntomodulin in the CNS: role of peripherally secreted and centrally produced peptides. Prog Neurobiol 2010;92(3):442-462.

14. Holst JJ. The physiology of glucagon-like peptide 1. Physiol Rev 2007;87(4):1409-1439.

15. Lovshin JA, Drucker DJ. Incretin-based therapies for type 2 diabetes mellitus. Nature Reviews Endocrinology 2009;5(5):262.

16. Batista AF, Forny-Germano L, Clarke JR, Lyra e Silva NM, Brito-Moreira J, Boehnke SE, et al. The diabetes drug liraglutide reverses cognitive impairment in mice and attenuates insulin receptor and synaptic pathology in a non-human primate model of Alzheimer's disease. J Pathol 2018;245(1):85-100.

17. Long-Smith CM, Manning S, McClean PL, Coakley MF, O'Halloran DJ, Holscher C, et al. The diabetes drug liraglutide ameliorates aberrant insulin receptor localisation and 
signalling in parallel with decreasing both amyloid- $\beta$ plaque and glial pathology in a mouse model of Alzheimer's disease. Neuromolecular medicine 2013;15(1):102-114.

18. Yang Y, Zhang J, Ma D, Zhang M, Hu S, Shao S, et al. Subcutaneous administration of liraglutide ameliorates Alzheimer-associated tau hyperphosphorylation in rats with type 2 diabetes. J Alzheimer's Dis 2013;37(3):637-648.

19. Qi L, Ke L, Liu X, Liao L, Ke S, Liu X, et al. Subcutaneous administration of liraglutide ameliorates learning and memory impairment by modulating tau hyperphosphorylation via the glycogen synthase kinase- $3 \beta$ pathway in an amyloid $\beta$ protein induced alzheimer disease mouse model. Eur J Pharmacol 2016;783:23-32.

20. Hansen HH, Barkholt P, Fabricius K, Jelsing J, Terwel D, Pyke C, et al. The GLP-1 receptor agonist liraglutide reduces pathology-specific tau phosphorylation and improves motor function in a transgenic hTauP301L mouse model of tauopathy. Brain Res 2016;1634:158-170.

21. Hansen HH, Fabricius $K$, Barkholt $P$, Kongsbak-Wismann $P$, Schlumberger $C$, Jelsing J, et al. Long-term treatment with liraglutide, a glucagon-like peptide-1 (GLP-1) Receptor agonist, has no effect on $\beta$-amyloid plaque load in two transgenic APP/PS1 mouse models of Alzheimer's disease. PloS one 2016;11(7):e0158205.

22. Villarroya $F$, Iglesias $R$, Giralt M. Retinoids and retinoid receptors in the control of energy balance: Novel pharmacological strategies in obesity and diabetes. Curr Med Chem 2004;11(6):795-805.

23. Goodman AB. Retinoid receptors, transporters, and metabolizers as therapeutic targets in late onset Alzheimer disease. J Cell Physiol 2006;209(3):598-603.

24. Mcilroy GD, Delibegovic M, Owen C, Stoney PN, Shearer KD, McCaffery PJ, et al. Fenretinide treatment prevents diet-induced obesity in association with major alterations in retinoid homeostatic gene expression in adipose, Liver, and hypothalamus. Diabetes 2013;62(3):825-836.

25. Morrice N, Mcilroy GD, Tammireddy SR, Reekie J, Shearer KD, Doherty MK, et al. Elevated Fibroblast growth factor 21 (FGF21) in obese, insulin resistant states is normalised by the synthetic retinoid Fenretinide in mice. Scientific Reports 2017;7:43782.

26. Preitner F, Mody N, Graham TE, Peroni OD, Kahn BB. Long-term Fenretinide treatment prevents high-fat diet-induced obesity, insulin resistance, and hepatic steatosis. Am J Physiol Endocrinol Metab 2009;297(6):E1420-E1429.

27. Shearer KD, Morrice N, Henderson C, Reekie J, Mcilroy GD, McCaffery PJ, et al. Fenretinide prevents obesity in aged female mice in association with increased retinoid and estrogen signaling. Obesity 2015;23(8):1655-1662.

28. Mody N, Agouni A, Mcllroy GD, Platt B, Delibegovic M. Susceptibility to diet-induced obesity and glucose intolerance in the APP SWE/PSEN1 A246E mouse model of Alzheimer's disease is associated with increased brain levels of protein tyrosine phosphatase 1B (PTP1B) and retinol-binding protein 4 (RBP4), and basal phosphorylation of S6 ribosomal protein. Diabetologia 2011;54(8):2143-2151.

29. Mody N, Graham T, Tsuji Y, Yang Q, Kahn BB. Decreased clearance of serum retinol binding protein and elevated levels of transthyretin in insulin-resistant ob/ob mice. American Journal of Physiology-Endocrinology and Metabolism 2008. 
30. Pfaffl MW. A new mathematical model for relative quantification in real-time RT-PCR. Nucleic Acids Res 2001;29(9):e45-e45.

31. Li Z, Liang $Y$, Xia N, Lai $Y$, Pan $H$, Zhou S, et al. Liraglutide reduces body weight by upregulation of adenylate cyclase 3. Nutr Diabetes 2017;7(5).

32. Baumeier C, Schlüter L, Saussenthaler S, Laeger T, Rödiger M, Alaze SA, et al. Elevated hepatic DPP4 activity promotes insulin resistance and non-alcoholic fatty liver disease. Molecular metabolism 2017;6(10):1254-1263.

33. Ghorpade DS, Ozcan L, Zheng Z, Nicoloro SM, Shen Y, Chen E, et al. Hepatocytesecreted DPP4 in obesity promotes adipose inflammation and insulin resistance. Nature 2018;555(7698):673.

34. Poungvarin N, Chang B, Imamura M, Chen J, Moolsuwan K, Sae-Lee C, et al. Genomewide analysis of ChREBP binding sites on male mouse liver and white adipose chromatin. Endocrinology 2015;156(6):1982-1994.

35. Herman MA, Peroni OD, Villoria J, Schön MR, Abumrad NA, Blüher $M$, et al. A novel ChREBP isoform in adipose tissue regulates systemic glucose metabolism. Nature 2012;484(7394):333.

36. Rufinatscha K, Radlinger B, Dobner J, Folie S, Bon C, Profanter E, et al. Dipeptidyl peptidase-4 impairs insulin signaling and promotes lipid accumulation in hepatocytes. Biochem Biophys Res Commun 2017;485(2):366-371.

37. Miyazaki M, Kato M, Tanaka K, Tanaka M, Kohjima M, Nakamura K, et al. Increased hepatic expression of dipeptidyl peptidase- 4 in non-alcoholic fatty liver disease and its association with insulin resistance and glucose metabolism. Molecular medicine reports 2012;5(3):729-733.

38. Röhrborn D, Wronkowitz N, Eckel J. DPP4 in diabetes. Frontiers in immunology 2015;6:386.

39. Liu J, Yang K, Yang J, Xiao W, Le Y, Yu F, et al. Liver-derived fibroblast growth factor 21 mediates effects of glucagon-like peptide- 1 in attenuating hepatic glucose output. EBioMedicine 2019.

40. Porter D, Kerr B, Flatt P, Holscher C, Gault V. Four weeks administration of Liraglutide improves memory and learning as well as glycaemic control in mice with high fat dietary-induced obesity and insulin resistance. Diabetes, Obesity and Metabolism 2010;12(10):891-899.

41. Sisley S, Gutierrez-Aguilar R, Scott M, D’Alessio DA, Sandoval DA, Seeley RJ. Neuronal GLP1R mediates liraglutide's anorectic but not glucose-lowering effect. J Clin Invest 2014;124(6):2456-2463.

42. Lee SA, Kim YR, Yang EJ, Kwon E-, Kim SH, Kang SH, et al. CD26/DPP4 levels in peripheral blood and T cells in patients with type 2 diabetes mellitus. J Clin Endocrinol Metab 2013;98(6):2553-2561.

43. Lamers D, Famulla S, Wronkowitz N, Hartwig S, Lehr S, Ouwens DM, et al. Dipeptidyl peptidase 4 is a novel adipokine potentially linking obesity to the metabolic syndrome. Diabetes 2011 Jul;60(7):1917-1925.

44. Clarke JR, Lyra E Silva NM, Figueiredo CP, Frozza RL, Ledo JH, Beckman D, et al. Alzheimer-associated Abeta oligomers impact the central nervous system to induce peripheral metabolic deregulation. EMBO Mol Med 2015 Feb;7(2):190-210. 
45. Vella A, Shah P, Reed A, Adkins A, Basu R, Rizza R. Lack of effect of exendin-4 and glucagon-like peptide-1-(7,36)-amide on insulin action in non-diabetic humans. Diabetologia 2002;45(10):1410-1415.

46. Chen L, Lyu J, Yang X, Ji W, Yuan B, Chen M, et al. Liraglutide ameliorates glycometabolism and insulin resistance through the upregulation of GLUT4 in diabetic KKAy mice. Int J Mol Med 2013;32(4):892-900.

47. Rolin B, Larsen MO, Gotfredsen CF, Deacon CF, Carr RD, Wilken M, et al. The longacting GLP-1 derivative NN2211 ameliorates glycemia and increases $\beta$-cell mass in diabetic mice. American Journal of Physiology-Endocrinology and Metabolism 2002;283(4):E745-E752.

48. Tamura K, Minami K, Kudo M, lemoto K, Takahashi H, Seino S. Liraglutide improves pancreatic beta cell mass and function in alloxan-induced diabetic mice. PLoS One 2015;10(5):e0126003.

49. Gil-Lozano M, Mingomataj EL, Wu WK, Ridout SA, Brubaker PL. Circadian secretion of the intestinal hormone GLP-1 by the rodent L cell. Diabetes 2014 Nov;63(11):36743685.

50. Gil-Lozano M, Wu WK, Martchenko A, Brubaker PL. High-fat diet and palmitate alter the rhythmic secretion of glucagon-like peptide- 1 by the rodent L-cell. Endocrinology 2015;157(2):586-599.

51. Brubaker PL, Gil-Lozano M. Glucagon-like peptide-1: The missing link in the metabolic clock? Journal of diabetes investigation 2016;7:70-75.

52. Ando H, Ushijima K, Fujimura A. Indirect effects of glucagon-like peptide-1 receptor agonist exendin-4 on the peripheral circadian clocks in mice. Plos one 2013;8(11):e81119 
Figure 1

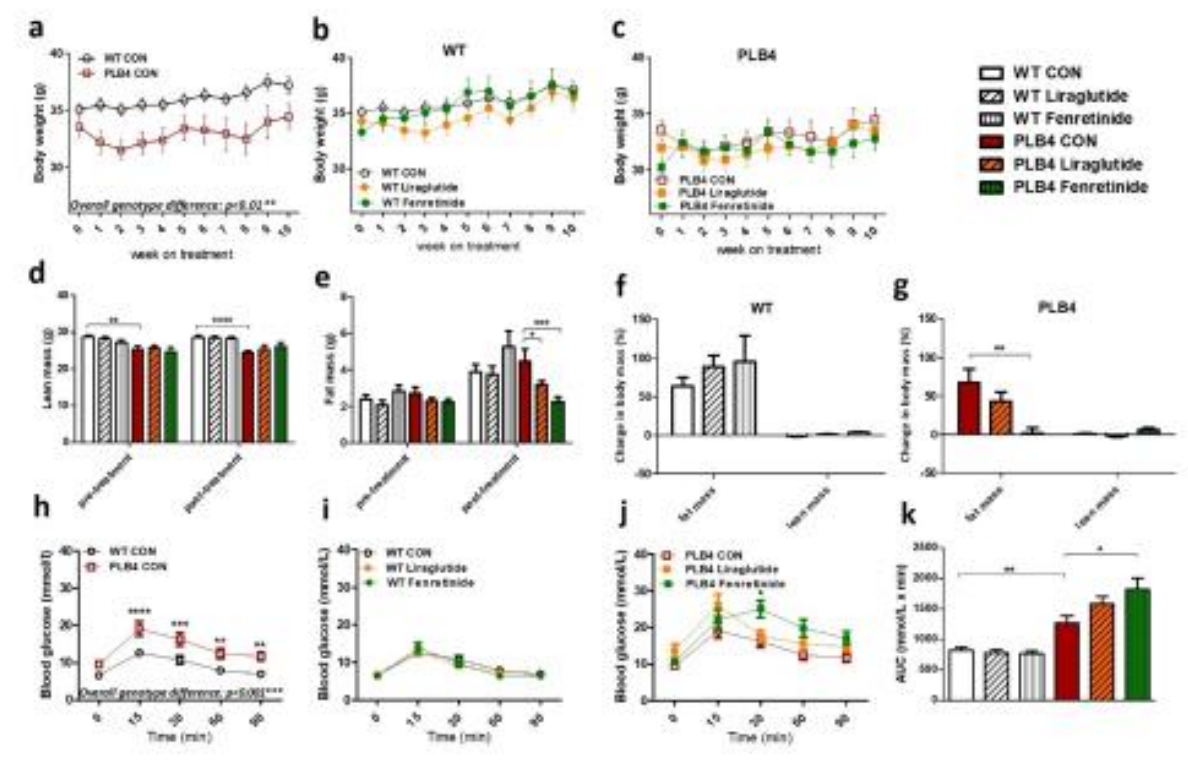


Figure 2

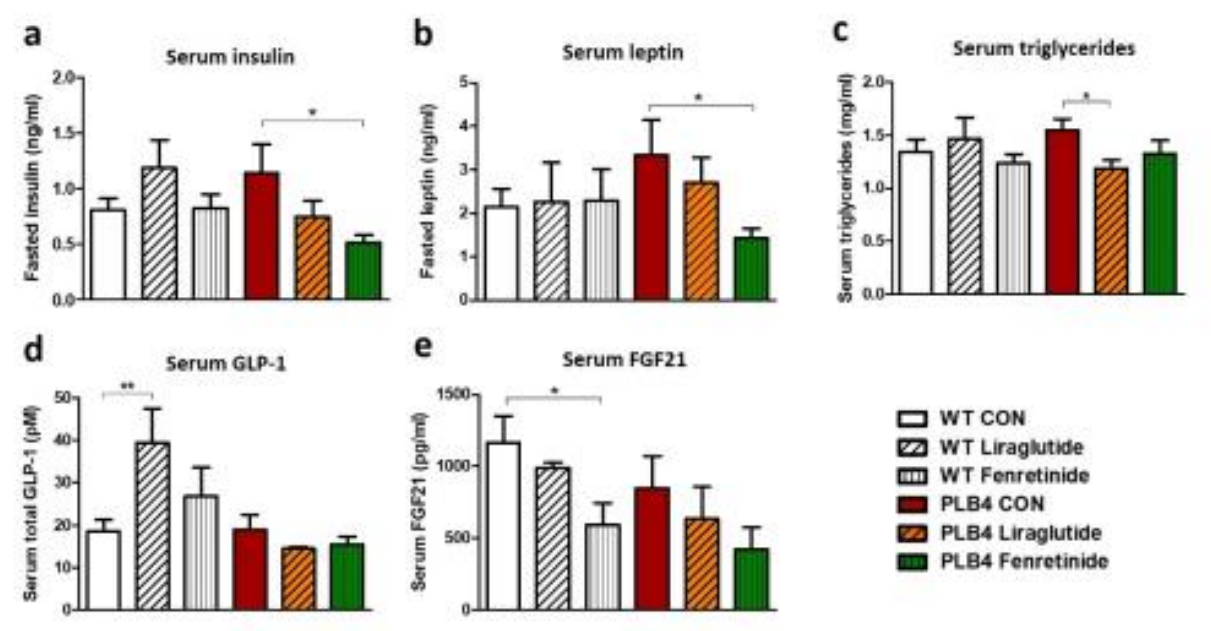


Figure 3

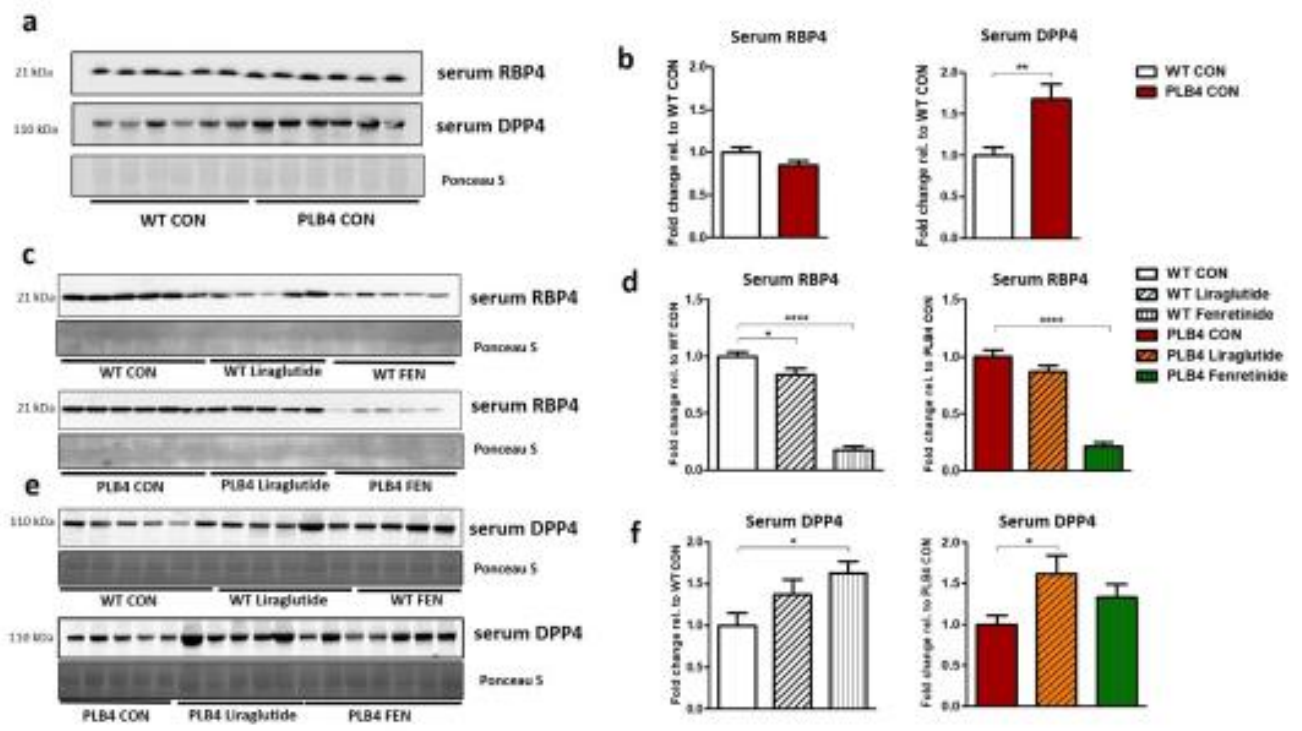


Figure 4
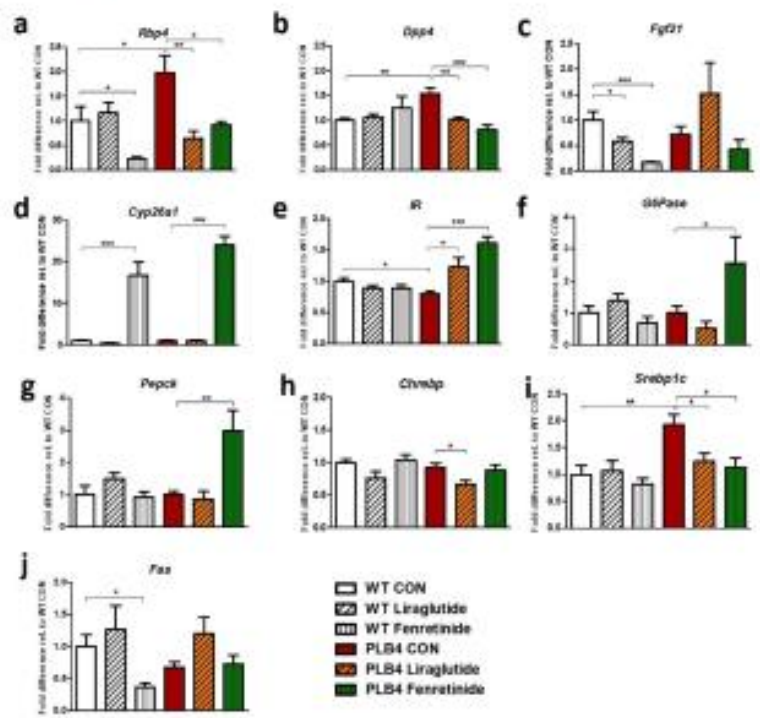

믄 CON

WT Liagutide
WT Fenresende

- PlBatcon

- PLBS fertratiabe 
Figure 5

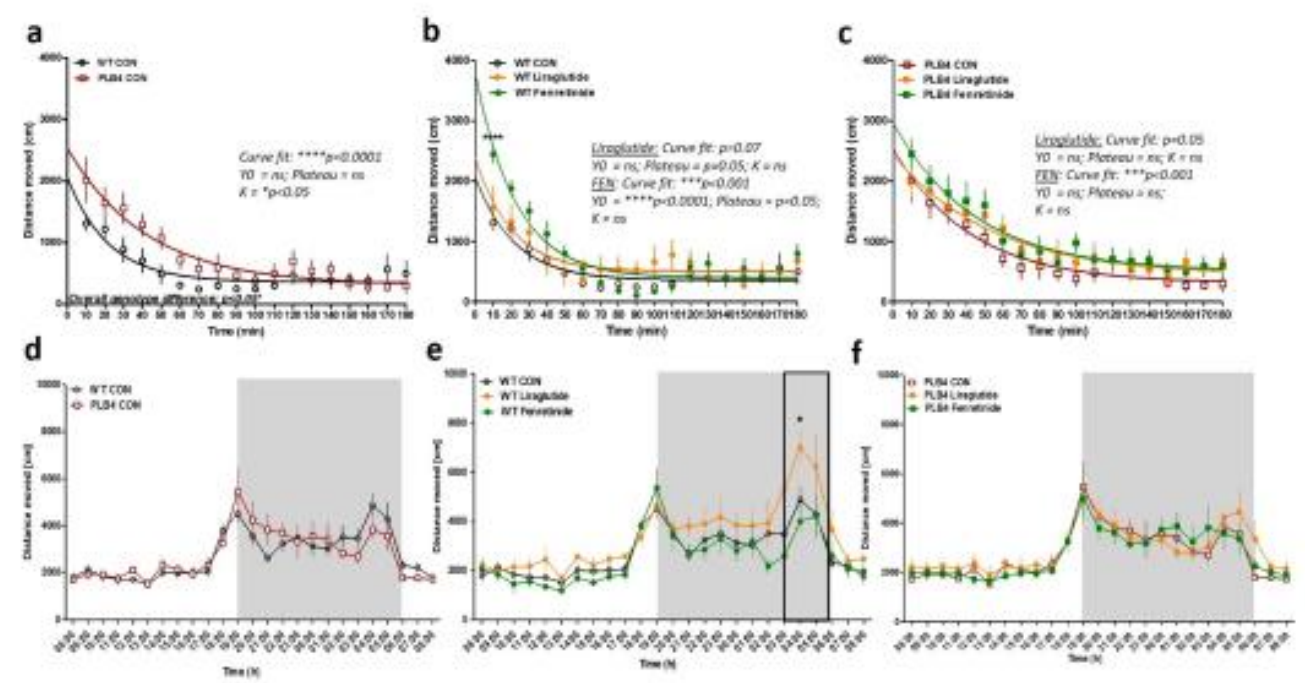




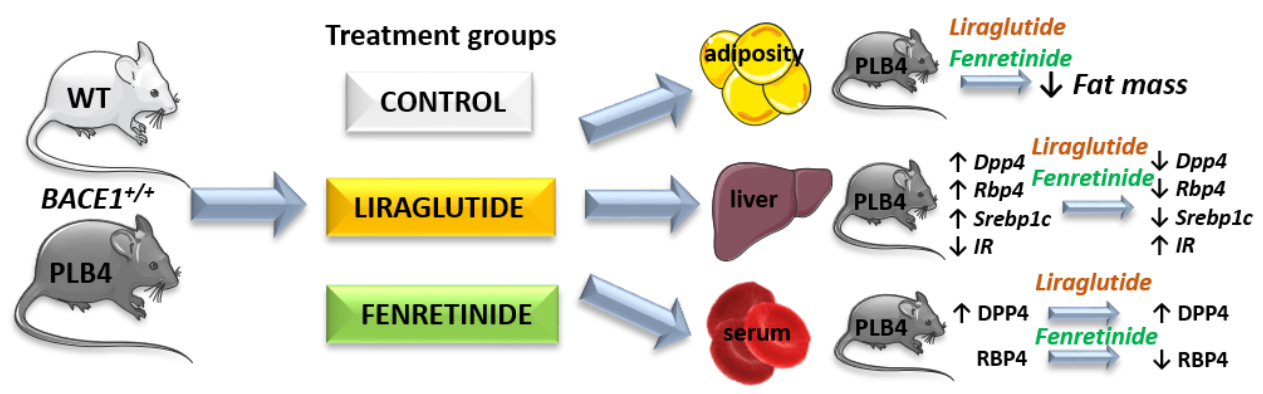

